\title{
Application of OpenLayers in marine information monitoring
}

\author{
HE Yuqin, BI Zhenbo,TIAN Haobin, DUAN Kai, WU Jiashuai, and WANG Hongwei \\ School of Mathematics, Physics \& Information Science, Zhejiang Ocean University, Zhoushan, Zhejiang 316022
}

\begin{abstract}
Marine information monitoring plays an important role in solving marine environment and controlling marine ecology. OpenLayers, as an open source development tool for WebGIS client, has the characteristics of small size and powerful function compared with commercial WebGIS software. This paper discusses the application of OpenLayers in ocean information monitoring based on OpenLayers. Firstly, The paper gives a basic overview of OpenLayers, then summarizes its related research, constructs the system framework for ocean information monitoring system and discusses the related key technologies. Finally, it studies the application of ocean information monitoring. From the research point of view, open source OpenLayers performs well, meets the general needs of marine information monitoring, and can significantly reduce development costs.
\end{abstract}

\section{Introduction}

With the in-depth development of national Marine economy, Marine information monitoring plays an vital role in people's understanding of Marine environment and management and control of Marine ecology. Currently, there are varieties of methods for Marine information monitoring, among which webgis-based monitoring is an important one. However, due to the high price and complex operation of WebGIS commercial tools, the researchers' development pace is often limited. However, in recent years, with the popularity of open source development, many open source tools have gradually appeared in the field of GIS application, such as QGIS, gvSIG, GRASS GIS, MapWindow, uDig and OpenLayers. Although their functions are not as powerful as those of commercial softwares, they still have enough functions for general tasks. With the support of many open source enthusiasts, these open source tools can be updated very quickly. In the open source software, OpenLayers as a tool for client development, in recent years has attracted the attention of numerous of users, compared with other WebGIS tools, it has the characteristics of small volume and powerful, in recent years have been applied in many fields, such as weather, sea ship management, emergency transportation, cultural relics management, logistics management, tourism management and remote fire management, etc. As an important WebGIS tool, there is no relevant research on Marine information monitoring at present. Therefore, this paper aims at Marine information monitoring and focuses on its application in this field, so as to provide some new ideas for the research and development of Marine information monitoring.

\section{OpenLayers Overview}

OpenLayers is a JavaScript class library package for WebGIS client development, which is used for network access of map data.As an open source project, it is designed to provide a powerful map display function for Internet clients, including map data display and related operations, with flexible extension mechanism.It is based on ActionScript and Flexb to redact, it can support various standard map services, such as WMS,WFS,WMTS,OSM,etc.As an open source and free client open source development framework, with better cross-platform capability. One of the vital advantages of OpenLayers in marine information monitoring is that it can make full use of HTML5 technology, give full play to the advantages of $\mathrm{H} 5$, adapt to the continuous improvement of client computer performance, and reduce the pressure of server-side page processing.

\section{Relevant Research}

As a powerful open source development framework, OpenLayers has characteristics of powerful function. It has attracted the attention of numerous researchers since its emergence. Schütze E takes OpenLayers as a free web map application example to discuss the technical status and potential of intelligent map browsing in Web browsers $^{[1]}$. Using tools such as OpenLayers, Henrie D describes the digital "planning" process available in the Scottish Ordnance Survey Historic Town Plan,avaliable on the website of the Scottish National Library ${ }^{[2]}$. Wisianto $A$ et al. integrated the pipeline data management application based on Web GIS application with Google map data set by using the open source technogy Sarp Map and OpenLayers ${ }^{[3]}$.Based on

\footnotetext{
* Corresponding author: bzb136@sina.com
} 
OpenLayers,Liu Hongyang et al. have studied the public meteorological service system. The analysis chart and road map have been superimposed by Open Layers GIS technology to complete the public meteorological service products meeting the need of users ${ }^{[4]}$. Based on OpenLayers and MongoDB, Huang Yixiu et al. studied the development of ship information management system. The system can display a large number of spatial data efficiently and synchronously in browser through OpenLayers, and provide map function services such as attribute query, spatial retrieval and statistical mapping ${ }^{[5]}$. Huang Chao has realized the basic functions of map enlargement, narrowing, translation and so on in the application of OpenLayers technology in the remote monitoring and control system of fire protection ${ }^{[6]}$. In the research of real-time visualization design and realization of tourism resource allocation based on Open Layers and Web Socket, Bao Shiliang et al. have realized the display of tourism resource allocation information on thematic tourism map by using Open Layers technology.Luo Quan used JS framework OpenLayers,GIS service software GeoServer and other open source technology to construct hydrologic on-line detection system ${ }^{[7]}$. Compared with commercial software, Luo Quan has better construction cost and operation and maintenance cost advantage in providing hydrological information monitoring service ${ }^{[8]}$.

\section{Marine Information Monitoring System}

Based on OpenLayers'related research in Section 2, the framework of marine information monitoring system we constructed according to the research objectives of this paper is shown in Fig.1. It should be noted that marine information monitoring is a very broad concept, with a wide range of objects, including important shipping routes or ports, sea water information, information on substances present in sea water, marine life information, seabed geological information, Marine climate information, estuarine and coastal zone information and ocean island information.But it mainly includes two kinds of information, marine environment and marine ecology, which have the characteristics of distribution area and location. Since the principle of client-side partial monitoring is the same, it is assumed that a few environmental elements and some important species (such as fish) monitoring in the marine environment will be taken as examples.

\subsection{System Framework}

For fig.1, there are three layers from top to bottom: front-end application layer, background logic layer and data layer.

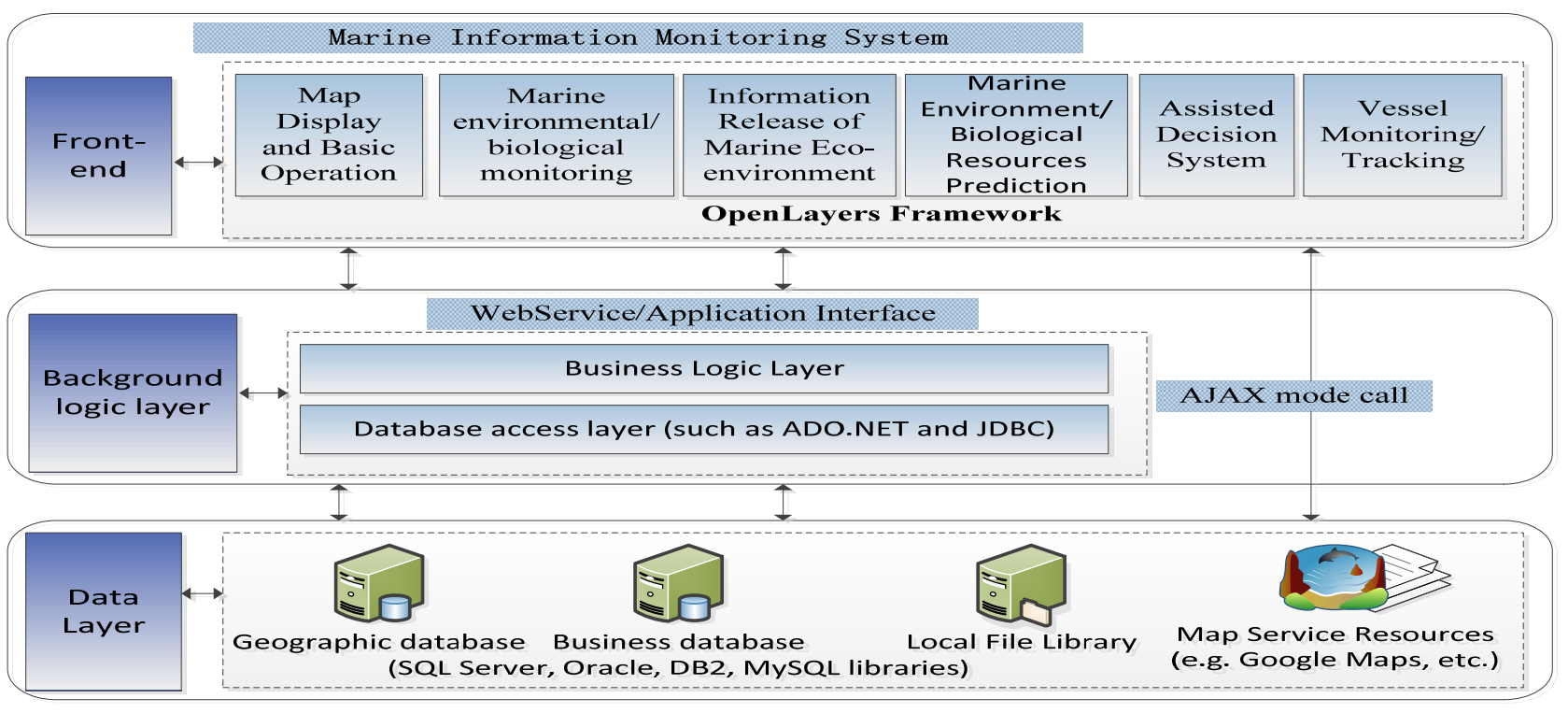

Fig. 1. Marine Information Monitoring System Framework

Front-end application layer mainly through OpenLayers map display and basic operations, sea area / marine environment (water temperature, salinity, $\mathrm{pH}$ value and chlorophyll, etc.) monitoring, marine life (large yellow croaker, and hairtail etc.), resource monitoring and marine environment / biological resource prediction are implemented by superposition of mapbased function layer and map service layer based on OpenLayers.

The background logic layer mainly realizes the business logic of marine information monitoring, this layer is mainly realized by WebService/Application Interface mode, and the core includes the business logic layer (marine environment monitoring, Marine life monitoring and marine environment / biological resources prediction, etc.) and database access layers (e.g. ADO.NET).

The data layer consists mainly of various types of database services, including geographic data, business data, knowledge, local files stored in various relational databases (SQL Server,Oracle,DB2,MySQL libraries) and other non-relational databases (such as MongoDB and Hbase, etc.). Map service resources, marine environment and ecological data, etc.

As shown in fig.1, OpenLayers plays an important role in the front-end application layer. 


\subsection{Key Technologies}

Through a careful analysis of the system architecture shown in fig.1, the key technologies involved include:

(1) Application Development Technology based on OpenLayers

OpenLayers is a JavaScript package for developing WebGIS clients. In the marine information monitoring projects developed with JavaScript pure client, OpenLayers is referred to as a function library, and the classes and properties and methods that it provides are invoked in the HTML document. So as to achieve Internet map publishing and functional operation ${ }^{[9]}$. OpenLayers classifies abstract transactions into classes, and its core classes includes Map,Layer,Source, and View, most actions are expanded around these core classes in order to implement map loading and related operations. The related application development technologies include HTML5 technology, CSS3 technology and JQuery technology, etc. They mainly assist OpenLayers to develop the foreground web application, and present the marine GIS function with the latest web technology.

(2) Construction of Mathematical Model for Marine Information Monitoring

Marine information includes marine ecology and marine environment. For marine environment and ecology, predicting, forecasting and changing trends should be specific, such as machine learning, the professional mathematical models of pattern recognition and data mining are used in marine environment and ecological analysis. The key task here is to develop mathematical tools or models that give accurate predictions, such as regression models (linear regression, nonlinear regression, Regression tree and rule-based model) and classification model (discriminant analysis and other linear classification models, nonlinear classification model, classification tree and rule-based model) and so on ${ }^{[10]}$. They are stored on the application server side in the form of class library, and expose the development interface for the foreground application program to call.

(3) Construction of thematic Database for all kinds of Business
The database is used as the foundation of marine information monitoring system, and all marine data information is stored and managed by database system to realize data sharing, reduce redundancy and duplication of data, and realize centralized control of data. Maintain data consistency and maintainability, this is the thematic database for all kinds of business. Specific construction objectives, need to build three types of data entities, namely, metadata, standard datasets and databases. Make it not only a local and regional source of information, but also serve the future construction of the "Intelligent Ocean". Taking "Intelligent Monitoring of important Biological Resources and Environment in Island Group Sea area"[11] as an example, the content of database construction should include: survey of seawater status in Island Group Sea area (sea water chemical elements such as $\mathrm{PH}$ value, suspended matter and dissolved oxygen, etc.). Investigation of hydrodynamic factors in island group sea area (such as water temperature, salinity, transparency of water color, about the background of the data in recent years, the representative of the month, etc.) and other marine life surveys ,etc.

\section{Application Development}

\subsection{Overview of Development}

On the basis of the previous analysis, the development of ocean information monitoring system involves foreground, background and application server. According to the emphasis of this article, this paper mainly discusses the development of foreground client. The system adopts the traditional software development life cycle method, through the top-down, step by step refinement of the structured software design method. The overall design framework of the system is shown in fig.2, which mainly consists of five modules, system login, basic functions, environmental factor management, prediction / forecast and background management, etc. The second and third module is completed under the environment of C\#, jQuery, OpenLayers 3 and html5, and so on. Login and site management based on ASP.NET, predict / forecast in the application serverside implementation.

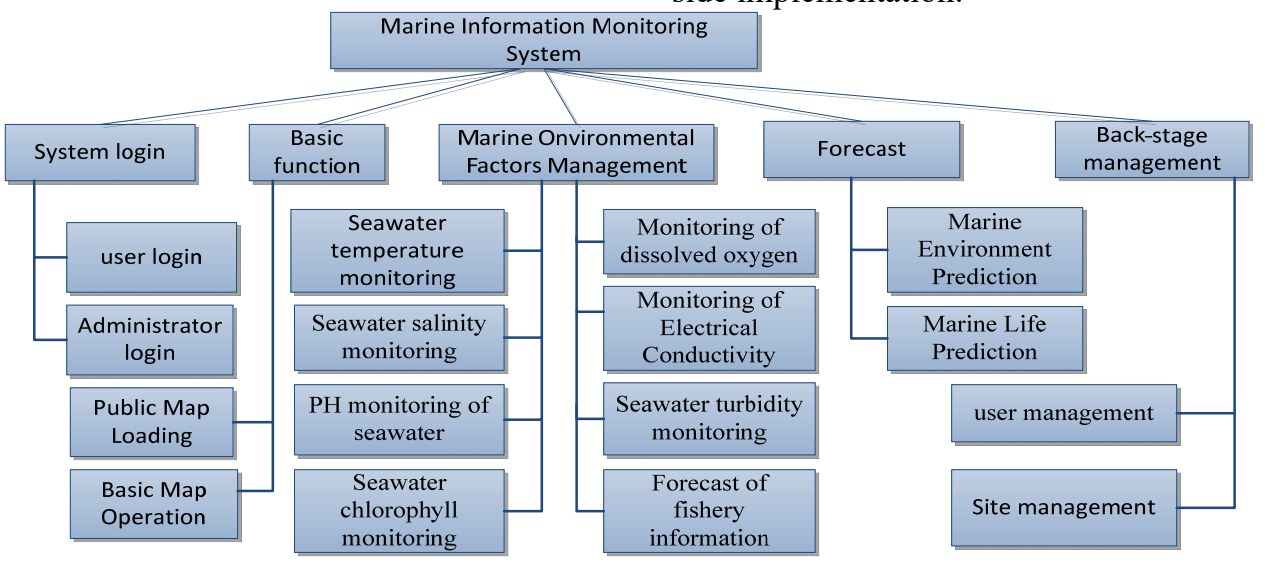

Fig. 2. System Function Diagram 


\subsection{Establishment of Important Mathematical Model for Ocean Monitoring}

In the research of the project in this paper, the model base is constructed to store various calculation models, to provide assistant decision-making for marine ecological restoration and protection, to provide evaluation and analysis for ecological restoration and protection effect, and to provide auxiliary decisionmaking service for government departments. The research targets include time analysis model, Markov chain analysis model, grey prediction model, principal component analysis model, association rule, BP neural network, support vector machine and system dynamics, etc.In order to maximize the use of software functions, these models are encapsulated in a class manner, ensuring the separation of application logic and core processing in marine monitoring, and making these models better for predicting fishery information in monitoring platforms, marine environment prediction, other marine life prediction, assessment and evaluation, marine information display and other application services.

\subsection{Data Organization Design}

In this system, two kinds of data are involved, namely geographic data and business data. According to the specific application, the two types of data are organized and designed, and the correlation between the data is shown in fig.3.

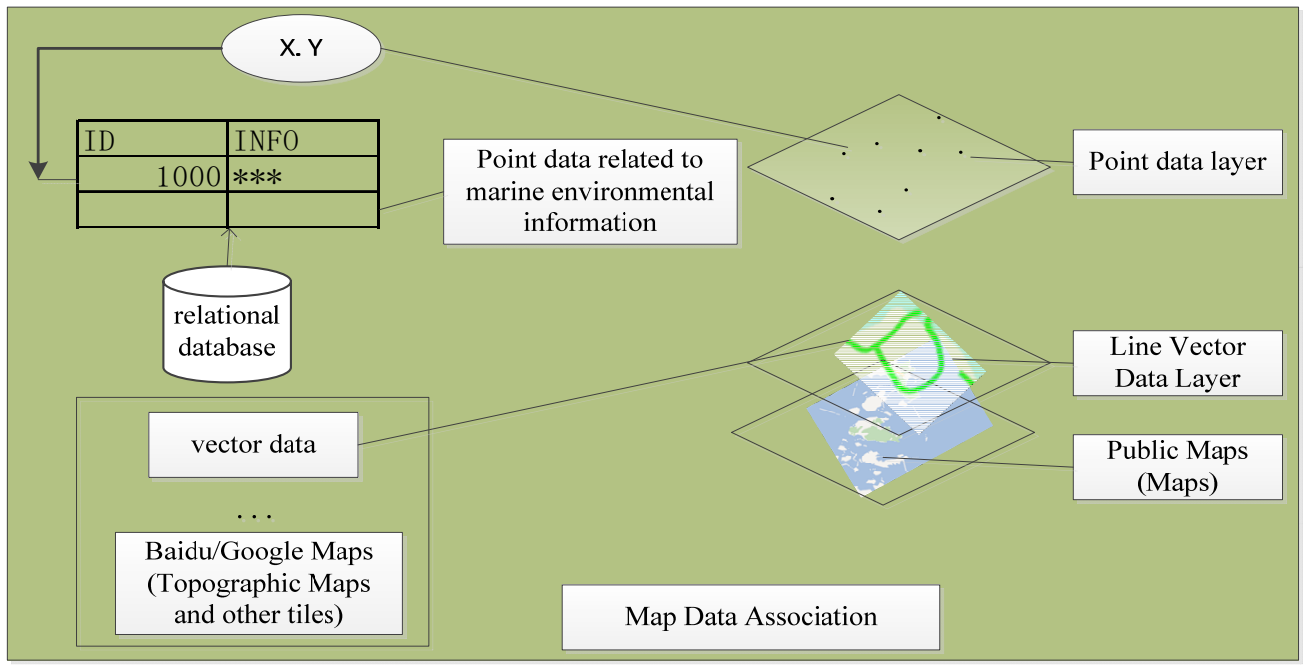

Fig. 3. Data Organization

For the geographic data, the system uses Google public map as the base map, and the upper layer superimposed the marine environment-related vector point data. For operational data, it mainly includes basic data (sources used to reflect monitoring data, mainly personnel, monitoring tasks, monitoring stations, monitoring areas, etc.), Marine eco-environmental monitoring data (including routine monitoring data and real-time monitoring data), Historical data (referred to all types of marine ecological and habitat data accumulated over a long period of time in the relevant sea areas) and metadata (In order to facilitate the management, application and maintenance of the marine monitoring information platform, a metadata base is established, which is used to explain and interpret the data sources and functions of various records.), etc. (fig.4).The system uses SQL Server2008 for storage. The system is based on MVC framework, the client sends the data service request to. Net server through AJAX, and the data is returned to the client in JSON format.

\subsection{System running}

According to the structure design and function design of the system, the system is developed based on VS2010.
According to the function module of the system, UI technology such as HTML5 and jQuery is used to build the system. After the system runs, it jumps to the login interface. Login is divided into ordinary user login and administrator login. Administrator login is mainly used in the background for user management and site management. The main applications of OpenLayers in the development of marine information monitoring include basic map loading, tile grid information display, map enlargement and reduction, map annotation, statistical display, map scale setting and multi-source data overlay display, etc. According to the map service requirement of WebGIS application system, OpenLayers meets the monitoring requirement of marine information client completely, and it is simple and quick to use. 


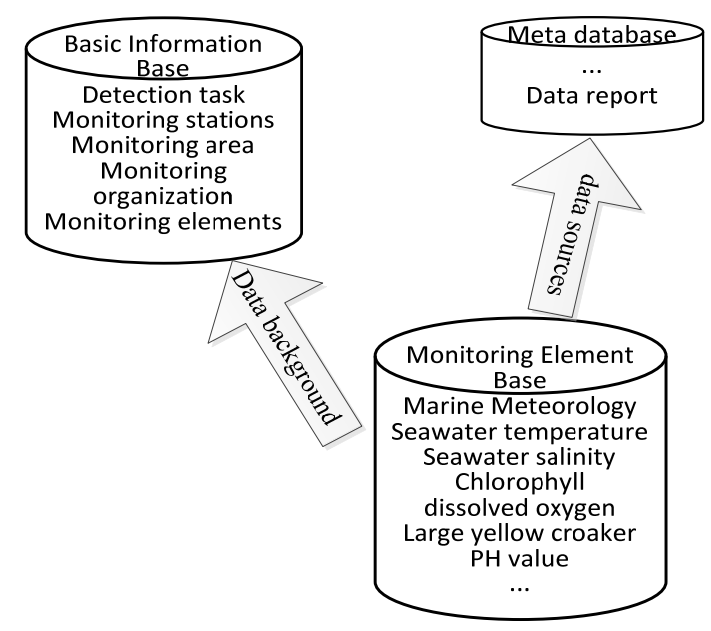

Fig. 4 The whole framework of marine information monitoring database

\section{Conclusions}

Ocean information monitoring is essential to marine ecosystems and global ecosystems. In recent years, marine biodiversity is being seriously threatened, mainly due to the destruction of the ecological environment of the ocean and coastal zone, overexploitation, pollution and inappropriate coastal aquaculture. The degradation of marine environment and the imbalance of marine ecology have affected the sustainable development of human society, especially the economic and social development of coastal areas. Therefore, it is of great significance to establish an effective monitoring system in time. This paper focuses on the application of OpenLayers in marine information monitoring. From the point of view of research, the open source OpenLayers performs well, accords with the general demand of marine information monitoring, and has potential application prospects.

\section{Acknowledgement}

This research was financially supported by Zhejiang cultural relics protection science and technology project (2017016), the National University Student Innovation and Entrepreneurship Training Program(201810340035) and Subprojects of Special Funds for Scientific Research in Marine Public Welfare Industry (201505025-6).

\section{References}

1. Schütze, E. "Current state of technology and potential of Smart Map Browsing in web browsers using the example of the Free web mapping application OpenLayers." Multimedia Technology Bremen University of Applied Sciences. Osnabrück/Germany, Bremen University of Applied Sciences. MSc in Computer Science 128 (2007).

2. Henrie, Daniel. "Ordnance Survey historic town plans of Scotland (1847-1895): Geo-referencing and web delivery with ArcIMS and OpenLayers." ePerimetron 4.1 (2009): 73-85.

3. Wisianto, Arie, Hidayatus Saniya, and Oki Gumilar. "Integrating Pipeline Data Management Application and Google Maps Dataset on Web Based GIS Application Using Open Source Technology Sharp Map and Open Layers." 2010 8th International Pipeline Conference. American Society of Mechanical Engineers, 2010.

4. Liu Hongyang, Lei Shengkai, Zhang Xiangfeng. "Application of OpenLayers in Public Meteorological Services." The 31st Annual Meeting of the Chinese Meteorological Society, the 4th Forum on the Development of Meteorological Services - Improving the Level of Hydrometeorological Disaster Prevention and Mitigation, and Promoting the Socialization of Meteorological Services in 2014.

5. Huang Yixiu, $\mathrm{Wu}$ Bin, $\mathrm{Lu}$ Zhenhua, et al. "Development of Ship Information Management System Based on OpenLayers and MongoDB." Geomatics \& Spatial Information Technology, 4 (2016): 147-149.

6. Huang Chao. Application of WebGIS technology in fire remote monitoring system[D]. Chengdu University of Technology, 2009.

7. Bao Shiliang, Zhou Weiqiang, Zhu Ruoxin, et al. Real-time visualization design and implementation of tourism resource allocation based on OpenLayers and WebSocket[J]. Geomatics \& Spatial Information Technology, 2017 (12): 122-125..

8. Luoquan. Design and implementation of hydrological online monitoring system integrated with WebGIS[D]. Guangxi University, 2015..

9. Guo Mingqiang, Huang Ying, Xie Zhong, etc. OpenLayers of WebGIS[M]. Beijing: Electronic Industry Press, 2016.

10. Max Kuhn, Kjell Johnson. Applied Prediction Modeling[M]. Beijing: Machinery Industry Press, 2016.

11. Yang Hua, Bi Zhenbo, Pan Hongjun, et al. "Research on the Framework of Intelligent Monitoring System for Important Biological Resources and Environment in the Sea Area of Islands Group." Marine Development and Management v.35; No.239.05 (2018): 67-72. 